\title{
In Memory of Professor Ismail Yazdi (1931-2020)
}

Mohammad Hossein Azizi, MD ${ }^{1 *}$

${ }^{1}$ Academy of Medical Sciences of Islamic Republic of Iran, Tehran, Iran

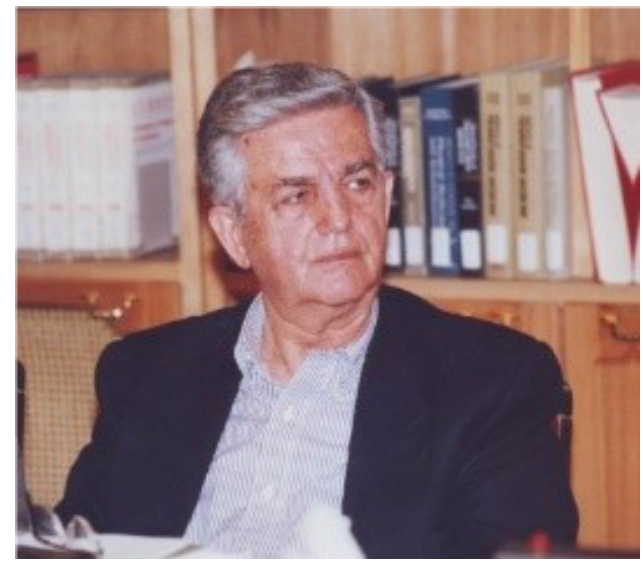

Professor Ismail Yazdi

It is with deep sorrow that we announce the passing away of Dr. Ismail Yazdi on 12th March 2020 in Pars Hospital, Tehran due to COVID 19. He was a distinguished professor of maxillofacial surgery and oral pathology at the Dentistry School of Tehran University. His demise is a great loss for the Iranian medical community.

He was born in Qazvin in northwest of Tehran, on April 25, 1931. After completing primary and high schools in Tehran in Adab School and at Dar-al Fonun, respectively, he entered Tehran University School of Dentistry and graduated as a dentist in 1952. He received a government-sponsored scholarship by Tehran University as the best graduate of that year and continued his post graduate training in the United States at Harvard and Tufts universities to study oral and maxillofacial surgery and oral pathology. ${ }^{1,2}$

On his return to Iran, as the first Iranian maxillofacial surgeon and oral pathologist, Professor Kamaleddin Armin (1914-1995), the chair of the department of pathology at Tehran University, invited him to join his department and establish the division of oral and maxillofacial pathology. Accordingly, with the help of Dr. Mohsen Sayyah, Dean of School of Dentistry and Professor Armin's support, the young energetic Dr. Yazdi founded the division of oral and maxillofacial pathology in Tehran University. In 1962, it became independent and joined the School of Dentistry and officially approved residency training was initiated. In due course, Dr. Yazdi became Dean of Tehran University
Dental School from 1969 to $1971 .^{2}$ He retired after more than four decades but continued his scientific and social activities until the end of his fruitful life. ${ }^{3}$ In summary, he played various effective academic, administrative and social roles.

Added to treatment of patients and performing maxillofacial operations, he taught many dentistry students and residents, and wrote over 60 scientific articles in domestic and international journals. The Journal of Oral Pathology and Oral Surgery was founded in 1965 by Professor Yazdi. He authored a text on oral and maxillofacial pathology for dental students and in

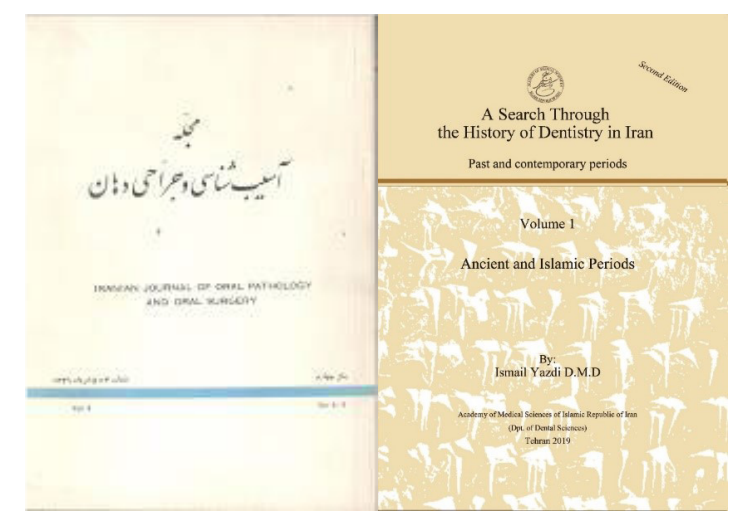

Left: Front Cover of Journal of Oral Pathology and Oral Surgery. Right: Back Cover of the Persian book entitled "A Search through the History of Dentistry in Iran".

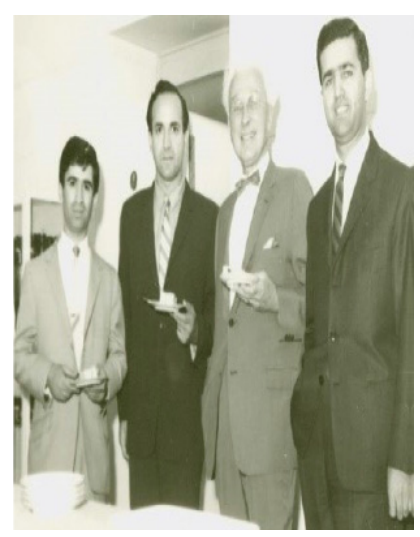

From left: Dr. Mirlouhi, Dr. Noparast, Gordan Agnew (Visiting Prof. from USA) and Prof. Yazdi, 1966-1967. 


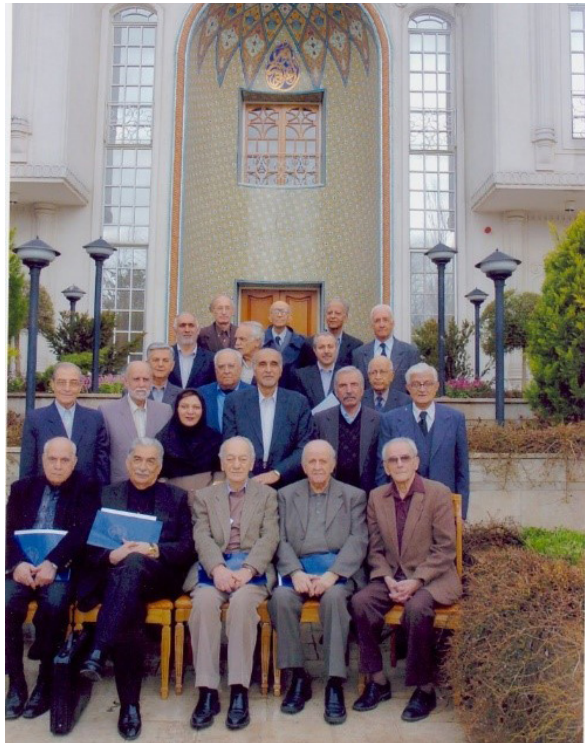

Professor Yazdi and his colleagues, Academy of Medical Sciences, TehranIran.

addition, he wrote a two-volume comprehensive book on the history of dentistry in Iran. He revised the second edition of the book before his death which is in press. He was one of the founders of Pars Hospital in Tehran. ${ }^{4}$

Professor Yazdi attended several international and local scientific meetings and delivered lectures. He was a consultant of the World Health Organization between 1972 and 1978. He was a member of the Iranian maxillofacial surgery and oral pathology board examination. Professor Yazdi and his colleagues founded the Iranian society of oral and maxillofacial pathologists in 1999.

He was an outstanding scientific figure in the Academy of Medical Sciences in Iran as a permeant member since
1992. He was also the director of the dentistry group of the academy between 1992 and 2020, as well as deputy for research between 2001 and 2005. Professor Yazdi was an influential member of the editorial board of the Archives of Iranian Medicine affiliated to the Academy of Medical Sciences of Iran, since its foundation in 1998. He was an active editor, and served as an expert peer reviewer. ${ }^{4}$

For his lifelong efforts, he received several awards including the first rank medal of culture in 1957 and the first rank national medal of science in 2008. Dr. Yazdi received the latter in recognition of his contributions to scientific progress in Iran. ${ }^{4}$ His memory will remain vibrant in the Iranian medical community.

\section{Conflict of Interest Disclosures}

None.

\section{Ethical Statement}

Not applicable.

\section{Acknowledgment}

I would like to thank Touraj Nayernouri MD, Neurosurgeon for his valuable comments and Miss Mahtab Nosrati for providing some photos.

\section{References}

1. Bahadori M, Eslami M, Azizi MH. A brief history of oral and maxillofacial pathology in Iran. Arch Iran Med. 2018; 21(11):551-5.

2. Azizi MH, Raees-Jalali GA, Noroozi H. A brief history of the publication of biomedical journals in Iran between 1901 and 1979. Arch Iran Med. 2009;12(2):204-11.

3. Official website of The School of Dentistry of Tehran University. Available from: http://dentistry.tums.ac.ir/fa/news/11810. Accessed March 18, .2020

4. Biography of Professor Yazdi. In: Yazdi I. A search through History of Dentistry in Iran. 2nd ed. Under Press; 2020 